\title{
Wholegrain food acceptance in young Singaporeans
}

\author{
J. E. Neo ${ }^{1}$ and I. A. Brownlee ${ }^{1}$ \\ ${ }^{1}$ Human Nutrition Research Centre, School of Agriculture, Food \& Rural Development, Newcastle University, \\ Singapore.
}

Epidemiological studies from around the world have consistently associated habitual consumption of whole grains with reduced disease and mortality risk ${ }^{(1)}$. The Singaporean public health agency (the Health Promotion Board) emphasise the importance of whole grain intake through recommendation of two to three servings of whole grains a day through collaboration with the food industry and out-of-home vendors to include whole grain alternatives ${ }^{(2)}$. Despite these recommendations, National Nutrition Survey (2004) shows that only $13.7 \%$ of Singaporeans consumed 0.5 serving or more of whole grains daily ${ }^{(3)}$. Previous consumer studies had determined potential barriers faced by individuals when attempting to incorporate whole grains into their diet ${ }^{(4)}$. Therefore, the primary objective of this study is to determine the knowledge of whole grains, salient barriers and the effectiveness of a whole grain acceptability trial whereby a variety of wholegrain products were given to participants.

Ethical approval was provided by the Newcastle University SAgE Faculty Ethics Committee for this study. A total of 30 participants, who were in their final year of university were selected to participate. The study comprised of two focus groups prior to and immediately following a 2-week wholegrain food acceptability trial. Barriers towards whole grain consumption and experiences of the acceptability trial were discussed during the semi-structured focus groups.

Potential barriers such as personal factors (relating to personal preference and wholegrain food knowledge), product-specific factors and external factors (influences affecting food purchasing decisions not governed by the individual) were identified with sensory and habitual being stronger barriers, as highlighted in Fig. 1. The wholegrain acceptability trial was not successful in altering the taste preference of the consumers but it did manage to increase acceptance of some of the wholegrain products.

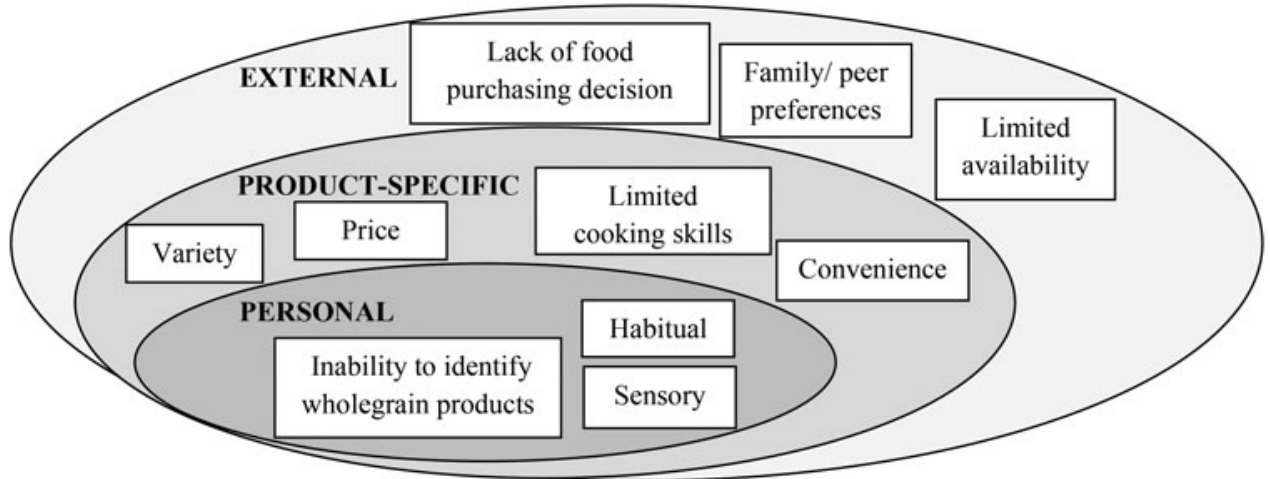

Fig. 1. Perceived barriers towards wholegrain consumption in young Singaporean adults

In conclusion, characterisation of such barriers to whole grain consumption could help develop population-specific strategies by public health agencies and food manufacturers to overcome these barriers.

1. Ye EQ, Chacko SA, Chou EL et al. (2012) J Nutr 142, 1304-1313

2. Health Promotion Board: "Whole-Grains The Wise Choice!" http://www.hpb.gov.sg/HOPPortal/health-article/6360 [accessed 28th March 2014]

3. Health Promotion Board (2004) Report of the National Nutrition Survey.

4. Kuznesof S, Brownlee IA, Moore C et al. (2012) Appetite 59, 187-193. 